\title{
No relevant pharmacokinetic drug-drug interaction between nintedanib and pirfenidone
}

\author{
Luca Richeldi ${ }^{1,2}$, Sophie Fletcher ${ }^{2,3}$, Huzaifa Adamali ${ }^{4}$, Nazia Chaudhuri ${ }^{3,5}$, \\ Sabrina Wiebe ${ }^{6}$, Sven Wind ${ }^{6}$, Kathrin Hohl ${ }^{6}$, Andrew Baker ${ }^{7}$, \\ Rozsa Schlenker-Herceg ${ }^{8}$, Susanne Stowasser ${ }^{9}$ and Toby M. Maher ${ }^{3,10}$
}

Affiliations: ${ }^{1}$ Università Cattolica del Sacro Cuore, Fondazione Policlinico A. Gemelli, Rome, Italy. ${ }^{2}$ Dept of Respiratory Medicine, University Hospital Southampton and Southampton NIHR Respiratory Biomedical Research Unit, University Hospital Southampton, Southampton, UK. ${ }^{3}$ Translational Research Collaboration Inflammatory Respiratory Disease Centre, Manchester, UK. ${ }^{4}$ Bristol Interstitial Lung Disease Service, North Bristol NHS Trust, Southmead Hospital, Bristol, UK. ${ }^{5}$ North West Lung Centre, Manchester University NHS Foundation Trust, Manchester, UK. ${ }^{6}$ Boehringer Ingelheim Pharma, Biberach, Germany. ${ }^{7}$ Boehringer Ingelheim, Bracknell, UK. ${ }^{8}$ Boeehringer Ingelheim Pharmaceuticals, Ridgefield, CT, USA. ${ }^{9}$ Boehringer Ingelheim International, Ingelheim am Rhein, Germany. ${ }^{10}$ National Institute for Health Research Respiratory Biomedical Research Unit, Royal Brompton and Harefield NHS Foundation Trust, and Fibrosis Research group, National Heart and Lung Institute, Imperial College, London, UK.

Correspondence: Luca Richeldi, Unità Operativa Complessa di Pneumologia, Università Cattolica del Sacro Cuore, Fondazione Policlinico A. Gemelli, Largo Agostino Gemelli 8, 00168 Rome, Italy. E-mail: luca.richeldidunicatt.it

@ERSpublications

There is no pharmacokinetic interaction between nintedanib and pirfenidone in patients with IPF http://ow.ly/utJD30msHeU

Cite this article as: Richeldi L, Fletcher S, Adamali H, et al. No relevant pharmacokinetic drug-drug interaction between nintedanib and pirfenidone. Eur Respir J 2019; 53: 1801060 [https://doi.org/10.1183/ 13993003.01060-2018].

ABSTRACT Nintedanib and pirfenidone are approved treatments for idiopathic pulmonary fibrosis (IPF). This open-label, two-group trial investigated the pharmacokinetic drug-drug interaction between these two drugs in patients with IPF.

Subjects not treated with antifibrotics at screening (group $1, n=20$ ) received a single nintedanib dose (150 mg) followed by pirfenidone (titrated to $801 \mathrm{mg}$ thrice daily) for 3 weeks, with a further single nintedanib dose $(150 \mathrm{mg}$ ) on the last day (day 23). Subjects treated with pirfenidone at screening (group 2, $\mathrm{n}=17$ ) continued to receive pirfenidone alone ( $801 \mathrm{mg}$ thrice daily) for 7 days, then co-administered with nintedanib (150 mg twice daily) for a further 7 days, before single doses of both treatments on day 16 .

In group 1, adjusted geometric mean (gMean) ratios (with/without pirfenidone) were $88.6 \%$ and $80.6 \%$ for nintedanib area under the plasma concentration-time curve (AUC) and maximum plasma concentration (Cmax), respectively. In group 2, gMean ratios (with/without nintedanib) were $97.2 \%$ and $99.5 \%$ for pirfenidone AUC and Cmax, respectively. For all parameters, the $90 \%$ confidence intervals included $100 \%$, suggesting similar exposure for administration alone and when co-administered. Both treatments were well tolerated.

These data indicate there is no relevant pharmacokinetic drug-drug interaction between nintedanib and pirfenidone when co-administered in IPF patients.

This article has supplementary material available from erj.ersjournals.com

Received: March 012018 | Accepted after revision: Oct 282018

This study is registered at ClinicalTrials.gov with identifier number NCT02606877. Researchers can use the link http:// trials.boehringer-ingelheim.com to find information in order to request access to the clinical study data, for this and other listed studies, after the submission of a research proposal and according to the terms outlined in the website. 


\section{Introduction}

Idiopathic pulmonary fibrosis (IPF) is a chronic, progressive, fibrosing form of interstitial lung disease of unknown aetiology resulting in progressive worsening of dyspnoea and lung function $[1,2]$. IPF has a poor prognosis and, until recently, no treatment for IPF was proven to be effective or approved for clinical use $[1,3]$.

In the past few years, two novel antifibrotic treatments, nintedanib and pirfenidone, have received regulatory approval in several countries after pivotal trials showed each was effective in slowing disease progression in patients with IPF $[4,5]$. Subsequently, both treatments have received positive recommendations in international evidence-based guidelines [6]. Given that nintedanib and pirfenidone are both effective at slowing disease progression, but neither halt nor reverse this ultimately fatal disease, there remains an unmet need for further therapeutic development [7].

With the availability of two antifibrotic drugs, and the existence of multiple signalling pathways involved in the pathogenesis of IPF, it is anticipated that combination therapy is likely to be a key focus of future IPF treatment development, similar to the management of several other chronic progressive diseases such as pulmonary arterial hypertension [2,7-11]. Although pirfenidone's mechanism of action has not been fully elucidated, it is thought to act upon several targets including glioma-associated oncogene homologue 2 -mediated transforming growth factor- $\beta$-triggered events [12-14]. Conversely, nintedanib acts on platelet-derived, fibroblast and vascular growth factor receptor tyrosine kinases to inhibit intracellular signalling $[15,16]$. This suggests that therapy with both compounds may offer additive or synergistic effects, resulting in greater clinical benefits than either treatment alone [7, 9]. However, there is some overlap in the tolerability profiles of pirfenidone and nintedanib in patients with IPF in terms of increases in liver enzymes and gastrointestinal events $[5,17,18]$. As combination therapy is a potential option for clinicians and patients, it follows that data on potential drug-drug interactions and the overall benefit/risk profile of combined nintedanib and pirfenidone treatment are urgently required in order to inform clinical decision-making [7].

Pirfenidone and nintedanib have different metabolic profiles. Pirfenidone is metabolised by various cytochrome P450 (CYP) enzymes, primarily CYP1A2 with minor contributions from CYP2C9, CYP2C19, CYP2D6 and CYP2E1, and predominantly excreted via the urine as the metabolite 5-carboxy-pirfenidone $[12,19]$. Co-administration of the antidepressant fluvoxamine, a strong inhibitor of CYP1A2 and other CYP isoenzymes, has been associated with a statistically significant increase in pirfenidone exposure, and pirfenidone is contraindicated in patients with concomitant use of fluvoxamine $[12,19]$. In contrast, the metabolism of nintedanib is via hydrolytic ester cleavage, resulting in the formation of the free acid moiety that is subsequently glucuronidated and excreted in the faeces [20]. Nintedanib has a low potential for drug-drug interactions via CYP enzymes [21].

At the time of planning this trial, nintedanib and pirfenidone combination treatment had only been tested in a phase II trial in Japanese patients with IPF [18]. This was a double-blind, randomised, placebo-controlled trial, in which 50 patients were treated with nintedanib on top of standard medical care with stratification according to pirfenidone use. The results indicated a trend towards lower nintedanib exposure in the presence of steady-state pirfenidone and raised the question of a potential pharmacokinetic drug-drug interaction. However, solid conclusions from the study could not be drawn, as the study design served a different purpose, exposure to nintedanib could only be compared between patient groups (inter-individually) and the number of evaluable pharmacokinetic observations $(<12$ per treatment group) was not deemed sufficient to assess the pharmacokinetic drug-drug interaction.

To provide further clarification, this study was designed to investigate the pharmacokinetic drug-drug interaction of nintedanib and pirfenidone when co-administered in individuals with IPF. The primary objective was to investigate the effects of steady-state pirfenidone on the pharmacokinetics of a single nintedanib dose, and to investigate the effect of steady-state nintedanib on the pharmacokinetics of pirfenidone at steady-state in patients with IPF. A secondary objective was to evaluate the safety of combination treatment.

\section{Material and methods}

This was an open-label, multi-dose, two-group trial in individuals with IPF (EudraCT 2015-000732-15; NCT02606877). Subjects were not randomised. Eligible individuals who were not on nintedanib or pirfenidone treatment at study entry were assigned to group 1, whereas those currently receiving full-dose pirfenidone entered group 2. Each group had a fixed-sequence design (figure 1). The study protocol was approved by an independent ethics committee (London-Surrey Borders Research Ethics Committee, London, UK) and competent authority (Medicines and Healthcare Products Regulatory Agency, London, UK). The study was conducted in accordance with the principles of the declaration of Helsinki and the 
a)

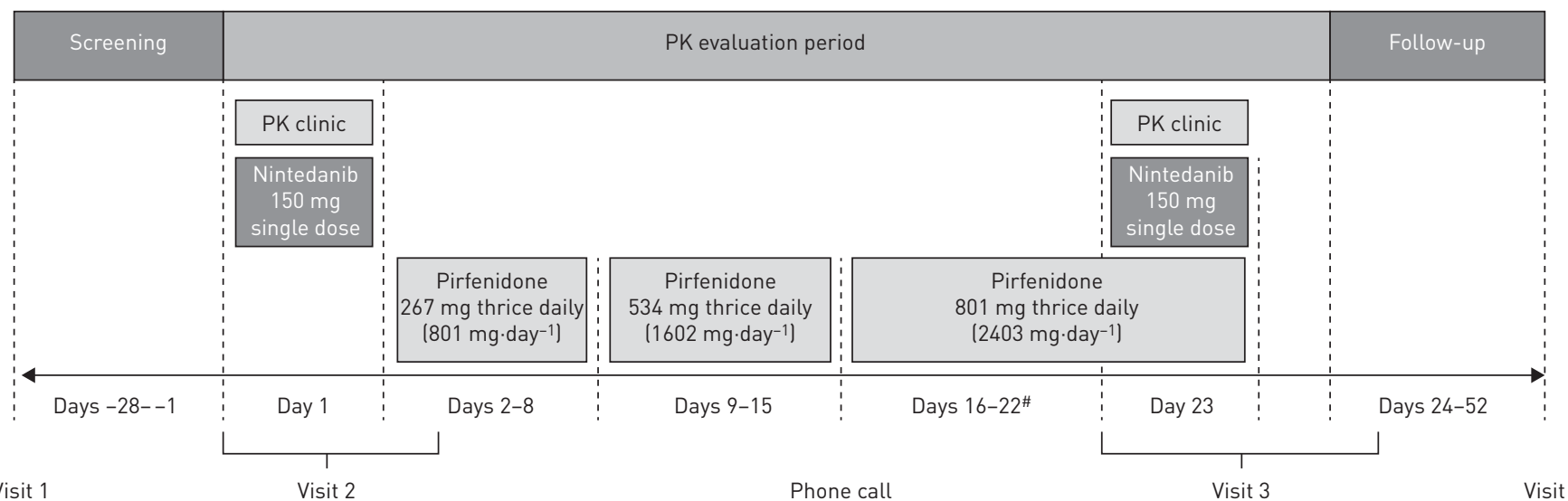

b)

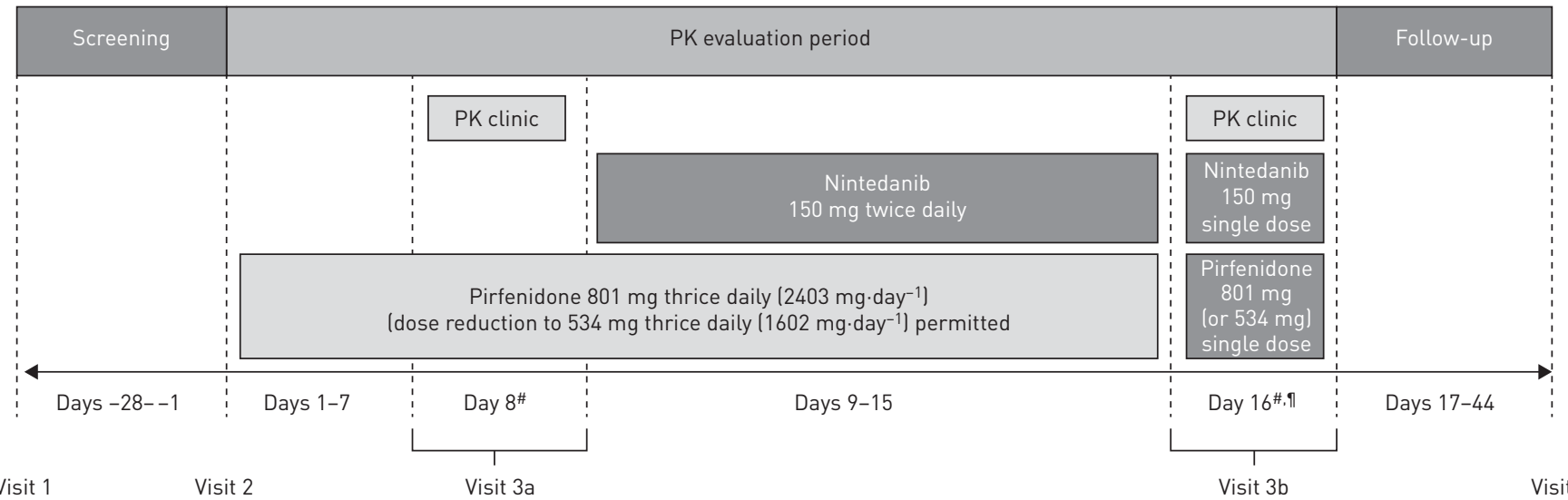

FIGURE 1 Study design. al group 1 ; b) group 2. PK: pharmacokinetic. \#: time windows were allowed to reach or to maintain the full pirfenidone dose (group 1: 6-day time window; group 2: two 3-day time windows) and to ensure stable 7-day treatment periods (groups 1 and 2: full-dose pirfenidone; group 2: combined treatment with nintedanib and pirfenidone); ${ }^{\uparrow}$ : on day 16 (visit 3b), subjects took single doses of pirfenidone $801 \mathrm{mg}$ and nintedanib $150 \mathrm{mg}$ only in the morning. PK sampling started $1 \mathrm{~h}$ prior to administration of single doses of pirfenidone and nintedanib and continued for $6 \mathrm{~h}$ post-drug administration. In group 1, PK samples were collected before and 0.5, 1, 2, 3, 4, 6, 8, 10 and $\sim 24 \mathrm{~h}$ after dosing on days 1 (nintedanib only) and 23. In group 2, PK samples were collected before and 0.5, 1, 2, 3, 4 and $\sim 6 \mathrm{~h}$ after dosing on days 8 (pirfenidone onlyl and 16 .

International Conference on Harmonisation Good Clinical Practice guidelines. Written informed consent was obtained from all subjects before study entry. The trial was undertaken at nine hospitals in the UK between May 2016 and March 2017.

\section{Subjects}

Subjects eligible for inclusion were men and women aged $\geqslant 40$ years, with IPF diagnosed according to current international guidelines [1] and a chest high-resolution computed tomography scan performed prior to screening. Suitable individuals were required to have a forced vital capacity $\geqslant 50 \%$ predicted and diffusing capacity of the lung for carbon monoxide $30-79 \%$ pred. Key exclusion criteria included elevated liver enzymes ( $>1.5$ times the upper limit of the normal range); increased bleeding risk (e.g. requiring therapeutic anticoagulation or high-dose antiplatelet therapy); current smoking; history of a thrombotic event within 12 months of screening; and severe renal impairment. Individuals who had previously received nintedanib in the past 2 weeks, had previous treatment with pirfenidone in the past 3 months (group 1 only) or had previously discontinued nintedanib or pirfenidone due to adverse events were excluded. Full exclusion criteria are provided in the supplementary material.

\section{Study design and treatments}

Figure 1 shows the study design and treatments received in the two groups. Group 1 received a single dose of nintedanib $150 \mathrm{mg}$ on day 1, pirfenidone (uptitrated from $267 \mathrm{mg}$ three times daily to $801 \mathrm{mg}$ three times daily) from days 2 to 23, with a single dose of nintedanib $150 \mathrm{mg}$ on the last day (day 23). Group 2 
received pirfenidone alone ( $801 \mathrm{mg}$ three times daily) for seven consecutive days (from day 1 to 8 ) and then co-administered with nintedanib $150 \mathrm{mg}$ twice daily for another seven consecutive days (from day 9 to 15), with single doses of pirfenidone $801 \mathrm{mg}$ and nintedanib $150 \mathrm{mg}$ on day 16 . A safety follow-up visit was arranged at least 28 days after last drug intake.

Nintedanib was supplied as $150 \mathrm{mg}$ soft gelatine capsules (Ofev; Boehringer Ingelheim Pharma, Biberach, Germany), and pirfenidone as $267 \mathrm{mg}$ hard capsules (Esbriet; Roche Pharma, Grenzach-Wyhlen, Germany). In both groups, oral doses of nintedanib and pirfenidone were administered with food. Treatment interruption and dose reductions were permitted for management of adverse events.

\section{Pharmacokinetic evaluation}

In group 1, venous blood samples for measurement of nintedanib and pirfenidone plasma concentrations were collected in potassium-EDTA-anticoagulant tubes up to $24 \mathrm{~h}$ after dosing on days 1 (nintedanib only) and 23 (figure 1). In group 2, blood samples for measurement of nintedanib and pirfenidone plasma concentrations were collected up to $6 \mathrm{~h}$ after dosing on days 8 (pirfenidone only) and 16. Plasma concentrations of nintedanib and pirfenidone (in the form of their free bases) were analysed using a validated liquid chromatography-mass spectrometry assay (Nuvisan, Neu-Ulm, Germany). The calibration curves for nintedanib and pirfenidone plasma in undiluted samples covered ranges of $0.05-50.0 \mathrm{ng} \cdot \mathrm{mL}^{-1}$ and $100-50000 \mathrm{ng} \cdot \mathrm{mL}^{-1}$, respectively.

\section{Safety evaluation}

The safety of nintedanib and pirfenidone was assessed by 12-lead ECG, vital signs (pulse, blood pressure), spirometry, routine laboratory assessments, physical examination, adverse event reporting and assessment of tolerability by the investigator. Details are provided in the supplementary material.

\section{Statistical and pharmacokinetic analysis}

The sample size determination was not based on a power calculation. A sample size of $\geqslant 12$ subjects with both evaluable pharmacokinetic assessments in each group was judged adequate to achieve the aims of this exploratory trial, in agreement with regulatory guidance [22]. Accounting for possible dropouts it was planned to recruit up to 34 subjects with the aim of entering 16 subjects in group 1 and 18 in group 2 .

Standard noncompartmental methods were used to calculate plasma pharmacokinetic parameters. area under the plasma concentration-time curve (AUC) was calculated using the log-linear trapezoidal rule from time zero (pre-dose) up to the time of the last quantifiable concentration (AUC0-tz) and extrapolated to infinity $(\mathrm{AUC} 0-\infty)$.

The aim of the study was to estimate 1) the relative bioavailability of nintedanib when administered alone or co-administered with pirfenidone in group 1; and 2) the relative bioavailability of pirfenidone when administered alone or co-administered with nintedanib in group 2. Pharmacokinetics were assessed using an intra-individual comparison, whereby treatment comparisons were made within subjects rather than between subjects, avoiding inter-subject variability [23]. The primary end-points in group 1 were

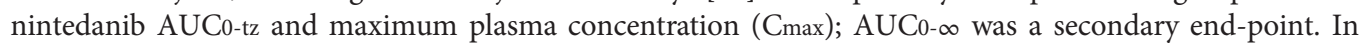
group 2, the primary end-points were steady-state pirfenidone AUC over a dosing interval $\tau$ (AUC $\tau, s s$ ) and Cmax at steady-state (Cmax,ss). The natural log-transformed AUC and Cmax values for nintedanib and pirfenidone were compared between groups using an ANOVA model. The primary analyses compared test treatment (nintedanib and pirfenidone co-administered) and reference treatment (mono-treatment with either nintedanib or pirfenidone) using the ANOVA model to evaluate the effect of treatment (fixed effect) and subjects (random effect) as sources of variation. This meant that individuals who had pharmacokinetic data for test and/or reference periods were used for the test/reference ratio estimate. For the pairwise comparison between the groups, the difference between the expected means was estimated by the difference in the corresponding least square means (point estimate); the $90 \%$ two-sided confidence intervals based on the t-distribution were also computed. These values were then back-transformed to the original scale to provide the point estimate (geometric mean) and interval estimates for the ratio of test and reference group. The main focus of the study was to estimate the magnitude of effects; as such, no hypothesis was tested and no equivalence range was specified. Sensitivity analyses were conducted based on the ANOVA model with treatment and subjects as fixed effects. This meant that only subjects with evaluable data in both treatment periods (test and reference) were used for the test/reference ratio estimate and $90 \%$ CIs, although all observations were entered into the model.

Other parameters were presented as descriptive statistics (arithmetic and geometric means (gMean), standard deviation and geometric coefficient of variation $(\mathrm{gCV})$ ). Noncompartmental analysis of plasma concentration-time data was performed using Phoenix WinNonlin (Pharsight Corporation, Cary, NC, USA). Statistical analyses were performed using SAS (SAS Institute, Cary, NC, USA). 


\section{Results}

Out of 51 screened subjects, 37 were eligible and entered the trial (figure 2). Of these, 20 entered group 1, and 17 entered group 2 (table 1). As expected, there were more males than females in the study and most individuals were white and aged $>60$ years. More than half of the subjects had smoked previously. In groups 1 and 2, $18(90.0 \%)$ and $15(88.2 \%)$ of patients had one or more concomitant diagnosis at baseline, respectively. The majority of patients in group $1(90.0 \%)$ and group $2(100.0 \%)$ took at least one concomitant therapy on-treatment. Further details of concomitant diagnoses and treatments are given in the supplementary material. All subjects entered into the trial were treated. In each group, 16 subjects completed trial medication and all of these completed a follow-up visit.

\section{Pharmacokinetics of nintedanib}

In group 1, 3 subjects prematurely discontinued trial medication due to adverse events and 1 for other reasons. Data from 17 subjects were evaluable for pharmacokinetic analysis of the reference treatment, with 12 of these evaluable for pharmacokinetic analysis of the test group on day 23. The summarised pharmacokinetic parameters are given in table 2.

In both treatment periods, absorption of nintedanib was relatively rapid, with median Cmax values reaching $26.1 \mathrm{ng} \cdot \mathrm{mL}^{-1}$ and $30.3 \mathrm{ng} \cdot \mathrm{mL}^{-1} \sim 2 \mathrm{~h}$ after administration with and without pirfenidone, respectively. Following peak absorption, plasma concentrations of nintedanib declined moderately fast until $\sim 6-10 \mathrm{~h}$, followed by a slower terminal phase (data not shown). The gMean terminal half-life of nintedanib was unaffected by co-administration with pirfenidone. Inter-individual variability in exposure was moderate to high for Cmax (gCV 65-103\%) and moderate for AUC (gCV 59-70\%) in both treatment periods.

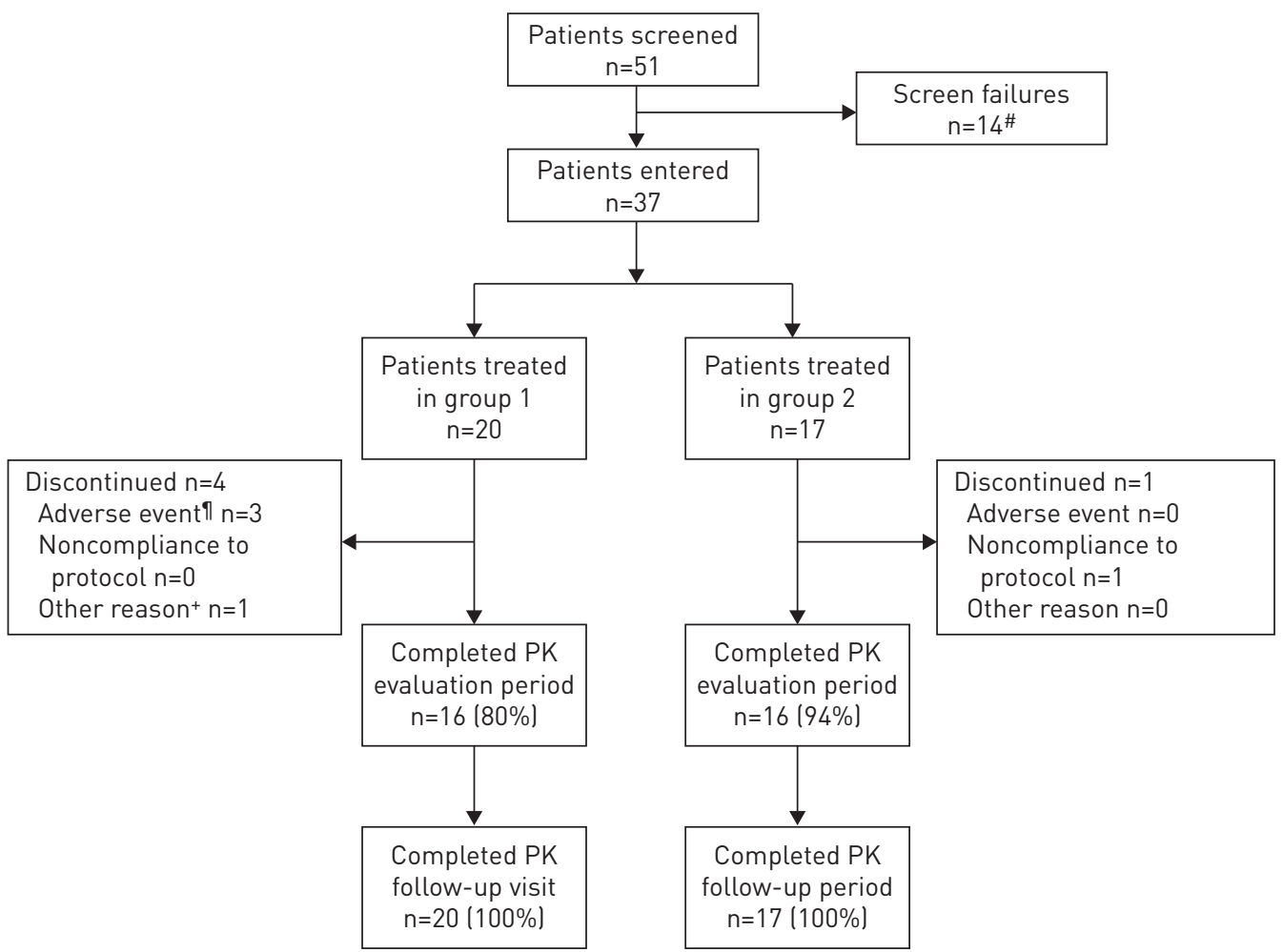

FIGURE 2 Participant disposition. A pharmacokinetic (PK) concentration or parameter collected from a patient was considered to be nonevaluable if, for example, a patient experienced emesis that occurred at or before two times median tmax (time to reach maximum observed plasma concentration ( $C_{\text {max }}$ )) of the respective treatment

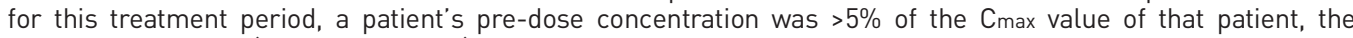
administered dose lamount of drugl was not in compliance with the clinical trial protocol, or restricted medication was used. In group 1, data from 17 subjects were considered evaluable for PK analysis of the reference treatment, with 12 of these evaluable for PK analysis of the test group on day 23. In group 2, data from 15 subjects were evaluable for PK analysis of the reference treatment, with 13 of these evaluable for PK analysis of the test group on day 23. In both treatment groups, 12 patients had evaluable PK data for the reference and the test treatment (included in the sensitivity analysis), respectively. ${ }^{\#}: 14$ (27.5\%) subjects were not enrolled, since they either did not meet the inclusion criteria or they did meet an exclusion criterion; " not due to worsening of disease under study; ${ }^{+}$: patient misunderstood the instructions for pirfenidone administration. 


\begin{tabular}{|c|c|c|}
\hline & Group 1 & Group 2 \\
\hline Subjects & 20 & 17 \\
\hline Male & 11 (55.0) & 15 (88.2) \\
\hline Age years & $71.5 \pm 7.8$ & $68.3 \pm 7.4$ \\
\hline$<65$ & $4(20.0)$ & 4 (23.5) \\
\hline$\geqslant 65$ to $<75$ & 11 (55.0) & $11(64.7)$ \\
\hline$\geqslant 75$ & $5(25.0)$ & $2(11.8)$ \\
\hline BMI $\mathrm{kg} \cdot \mathrm{m}^{-2}$ & $28.4 \pm 4.5$ & $28.0 \pm 3.4$ \\
\hline \multicolumn{3}{|l|}{ Race } \\
\hline White & 18 (90.0) & $16(94.1)$ \\
\hline Asian & $2(10.0)$ & $1(5.9)$ \\
\hline \multicolumn{3}{|c|}{ Time since IPF diagnosis years } \\
\hline$\leqslant 1$ & 17 (85.0) & $4(23.5)$ \\
\hline$>1$ to $\leqslant 3$ & $2(10.0)$ & $7(41.2)$ \\
\hline$>3$ to $\leqslant 5$ & $1(5.0)$ & $3(17.6)$ \\
\hline$>5$ & 0 & $3(17.6)$ \\
\hline \multicolumn{3}{|l|}{ Smoking status } \\
\hline Former & $14(70.0)$ & 9 (52.9) \\
\hline Never & $6(30.0)$ & $8(47.1)$ \\
\hline DLco \% predicted ${ }^{\pi}$ & $49.6 \pm 10.2$ & $43.3 \pm 9.5$ \\
\hline
\end{tabular}

Data are presented as $\mathrm{n}$, mean \pm SD or $\mathrm{n}(\%)$. BMI: body mass index; IPF: idiopathic pulmonary fibrosis; $D \mathrm{Lco}$ : diffusing capacity of the lung for carbon monoxide. \#: in treated population; ${ }^{\text {: }}$ corrected for haemoglobin at baseline.

A graphical representation of the individual and gMean values for the primary end-points shows no clear trend when nintedanib was given alone or co-administered with pirfenidone (figure 3 ). Comparing the individual exposure estimates in the two treatment periods, two subjects had a more pronounced decrease from reference to test, three showed a more pronounced increase from reference to test, and all other individuals showed comparable exposure estimates. Three subjects with the highest individual Cmax values in the reference period had no value in the test period. These subjects did not appear to have any noteworthy demographic characteristics at baseline compared with other individuals.

TABLE 2 Pharmacokinetic parameters of nintedanib after single oral administration of $150 \mathrm{mg}$ nintedanib alone (reference), and together with steady-state pirfenidone $801 \mathrm{mg}$ three times daily (test) ${ }^{\#}$

\begin{tabular}{|c|c|c|c|}
\hline \multicolumn{2}{|c|}{ Nintedanib $150 \mathrm{mg}$} & \multirow{2}{*}{$\begin{array}{c}\text { Adjusted" }^{\text {gMean ratio }} \\
\%(90 \% \mathrm{CI})\end{array}$} & \multirow{2}{*}{$\begin{array}{c}\text { Intra-individual } \\
\text { gCV } \%\end{array}$} \\
\hline $\begin{array}{c}\text { Alone } \\
\text { (reference) }\end{array}$ & $\begin{array}{l}\text { + pirfenidone } \\
801 \mathrm{mg} \text { (test) }\end{array}$ & & \\
\hline
\end{tabular}

\begin{tabular}{|c|c|c|c|c|}
\hline Subjects $n$ & 17 & 12 & & \\
\hline \multicolumn{5}{|l|}{ Primary end-points } \\
\hline $\mathrm{AUC} 0-\mathrm{tz} \mathrm{ng} \cdot \mathrm{h} \cdot \mathrm{mL}^{-1}$ & 169.7 & 150.3 & $88.6(65.4-120.0)$ & 45.1 \\
\hline $\mathrm{C}_{\max } \mathrm{ng} \cdot \mathrm{mL}^{-1}$ & 28.6 & 23.0 & 80.6 (51.3-126.8) & 74.6 \\
\hline $\mathrm{AUC} \mathrm{C}_{-\infty} \mathrm{ng} \cdot \mathrm{h} \cdot \mathrm{mL}^{-1}$ & 195.6 & 175.0 & 89.5 (66.3-120.7) & 44.3 \\
\hline \multicolumn{5}{|l|}{ Other end-points } \\
\hline$t \max h$ & $2.0(0.5-6.0)$ & $2.5(2.0-6.0)$ & & \\
\hline AUC $0-t z \mathrm{ng} \cdot h \cdot \mathrm{mL}^{-1}$ & 169.7 & 162.7 & $95.9(69.9-131.5)^{\S}$ & 45.1 \\
\hline $\mathrm{C}_{\max } \mathrm{ng} \cdot \mathrm{mL}^{-1}$ & 28.6 & 26.5 & $92.8(56.8-151.3)^{\S}$ & 75.0 \\
\hline $\mathrm{AUC}_{0-\infty} \mathrm{ng} \cdot \mathrm{h} \cdot \mathrm{mL}^{-1}$ & 195.6 & 190.7 & $97.5(71.7-132.5)^{\S}$ & 43.8 \\
\hline
\end{tabular}



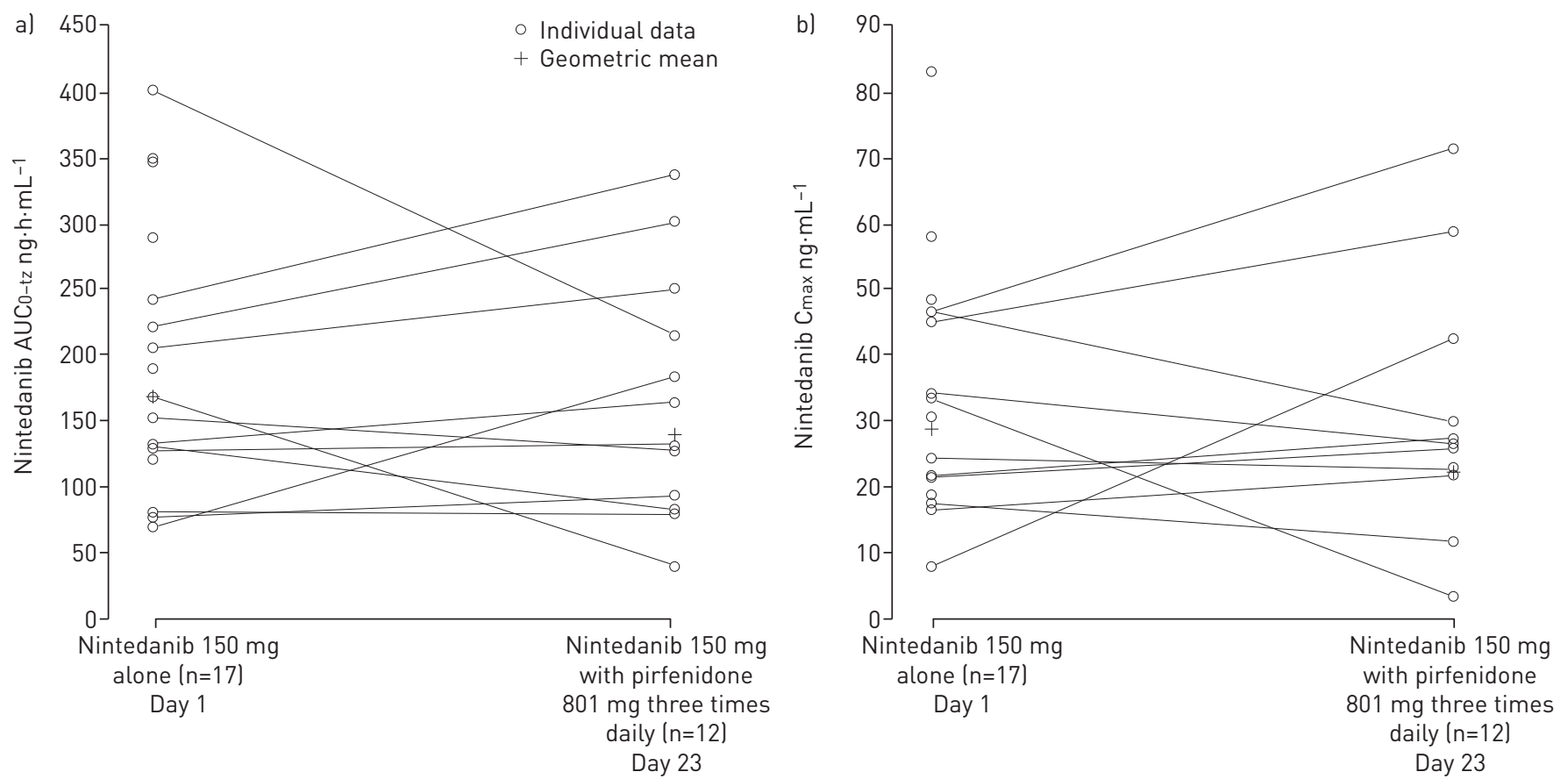

FIGURE 3 Comparison of individual and geometric mean nintedanib a) area under the plasma concentration-time curve from time 0 to the time of the last quantifiable data point ( $A \cup \mathrm{C}_{0}-\mathrm{tz}$ ) and b) maximum concentration in plasma ( $\mathrm{C}_{\max }$ ) after single oral administration of nintedanib $150 \mathrm{mg}$ alone, and together with pirfenidone $81 \mathrm{mg}$ thrice daily.

The point estimates for adjusted gMean ratios of nintedanib exposure when co-administered with pirfenidone versus administration alone were $80.6 \%, 88.6 \%$ and $89.5 \%$ for Cmax, AUC0-tz and AUC0- $\infty$, respectively (table 2). For all parameters, the $90 \%$ confidence intervals included $100 \%$, suggesting a similar exposure for administration alone and when co-administered with pirfenidone. The intraindividual variability was moderate to high ( $\mathrm{gCV} 44-75 \%$ ), which was reflected by the wide $90 \%$ confidence intervals.

A sensitivity analysis that considered only the 12 subjects with evaluable pharmacokinetic data in both treatment periods showed point estimates for the gMean ratios of nintedanib exposure of $92.8 \%, 95.9 \%$ and $97.5 \%$ for Cmax, AUC0-tz and $\mathrm{AUC} 0-\infty$, respectively.

\section{Pharmacokinetics of pirfenidone}

In group 2, one patient prematurely discontinued trial medication and one was not evaluable during the reference treatment period due to protocol non-compliance. Data from 15 subjects were included in the pharmacokinetic analysis of the reference treatment with 13 subjects included in the test group. The summarised pharmacokinetic parameters are given in table 3.

Steady-state gMean plasma concentration-time profiles of pirfenidone after multiple dosing before and after concomitant multiple dosing with nintedanib were comparable between the treatment groups (data not shown). Pirfenidone plasma levels peaked at $\sim 1 \mathrm{~h}$ and then declined rapidly and steadily throughout the dosing interval.

The extent of pirfenidone exposure as indicated by Cmax,ss and $A U \tau$,ss was comparable whether pirfenidone was administered alone or in the presence of nintedanib. In line with this, no trend towards increased or decreased exposure could be delineated from the comparison of individual and gMean Cmax,ss or AUC $\tau$,ss values (figure 4).

The point estimates for adjusted gMean ratios of pirfenidone exposure when co-administered with nintedanib (both at steady-state) versus pirfenidone alone were $97.2 \%$ for AUC $\tau$,ss and $99.5 \%$ for Cmax,ss (table 3). The corresponding $90 \%$ CIs covered $100 \%$ and were within the standard $80-125 \%$ bioequivalence acceptance range for $\mathrm{AUC}_{\tau, \mathrm{ss}}(88-108 \%)$ and for $\mathrm{Cmax}_{\text {,ss }}$ (88-113\%). Intra-individual variability was low (gCV values between $14.1 \%$ and $17.4 \%$ ). A sensitivity analysis that considered only the 12 subjects with evaluable pharmacokinetic data in both groups showed similar results. 
TABLE 3 Pharmacokinetic parameters of pirfenidone after multiple oral administration of 801 mg pirfenidone three times daily alone (reference), and together with steady-state nintedanib $150 \mathrm{mg}$ twice daily (test) ${ }^{\#}$

\begin{tabular}{cc} 
Pirfenidone $801 \mathrm{mg}$ three times daily \\
\hline $\begin{array}{c}\text { Alone } \\
\text { (reference) }\end{array}$ & $\begin{array}{l}\text { nintedanib } 150 \mathrm{mg} \\
\text { twice daily (test) }\end{array}$
\end{tabular}

\section{Adjusted" gMean ratio ${ }^{+}$ $\%(90 \% \mathrm{CI})$}

Intra-individual gCV \%

\section{Subjects $\mathbf{n}$ \\ Primary end-points \\ $\mathrm{AUC}_{\tau, \mathrm{ss}} \mathrm{ng} \cdot \mathrm{h} \cdot \mathrm{mL}^{-1}$ \\ $C_{\text {max,ss }} \mathrm{ng} \cdot \mathrm{mL}^{-1}$ \\ Other end-points \\ tmax,ss $h$ \\ Sensitivity analysis \\ $A U C_{\tau, s s} \mathrm{ng} \cdot \mathrm{h} \cdot \mathrm{mL}^{-1}$ \\ $\mathrm{C}_{\text {max,ss } \mathrm{ng} \cdot \mathrm{mL}^{-1}}$}

15

40200

10600

$1.00(0.5-3.0)$

40500

10700
13

39000

10500

$97.2(87.8-107.5)$

14.1

$99.5(87.9-112.6)$

17.4

$1.00(0.5-4.0)$

38500

10400
$95.2(85.9-105.5)^{\S}$

$97.2(85.6-110.4)^{8}$
14.1

17.5

Data are presented as adjusted gMean or median (range). gMean: geometric mean; gCV: geometric coefficient of variation; AUC $\tau$, ss: area under the plasma concentration-time curve over a dosing interval $\tau$ at steady-state; Cmax,ss: maximum measured plasma concentration at

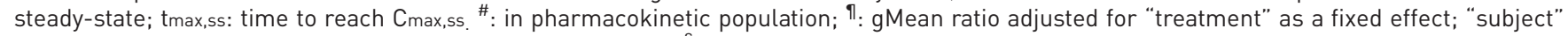
was considered as a random effect; ${ }^{+}$: ratio of test/reference; ${ }^{\S}$ : gMean ratio adjusted for "treatment" and "subject" as fixed effects.

\section{Safety}

In the two groups, 29 (78.4\%) subjects reported one or more adverse events (table 4). The most common adverse events were diarrhoea, nausea, lower respiratory tract infections, vomiting, headache and dyspepsia. Diarrhoea was the most frequently reported adverse event in both groups (group $130.0 \%$; group 2 23.5\%). Nausea and vomiting were reported with a frequency $>10 \%$ in each group. All reported adverse events were considered by the investigator to be mild or moderate apart from two cases of severe intensity in group 1 . One of these subjects treated with pirfenidone $267 \mathrm{mg}$ threes time daily felt weak and giddy. The patient interrupted treatment at the time of the event and later permanently discontinued pirfenidone. Another subject treated with pirfenidone $267 \mathrm{mg}$ three times daily reported a tooth infection of severe intensity. Neither of these two events was considered drug-related by the investigator and both subjects recovered from their events. All diarrhoea, nausea and vomiting events were National Cancer

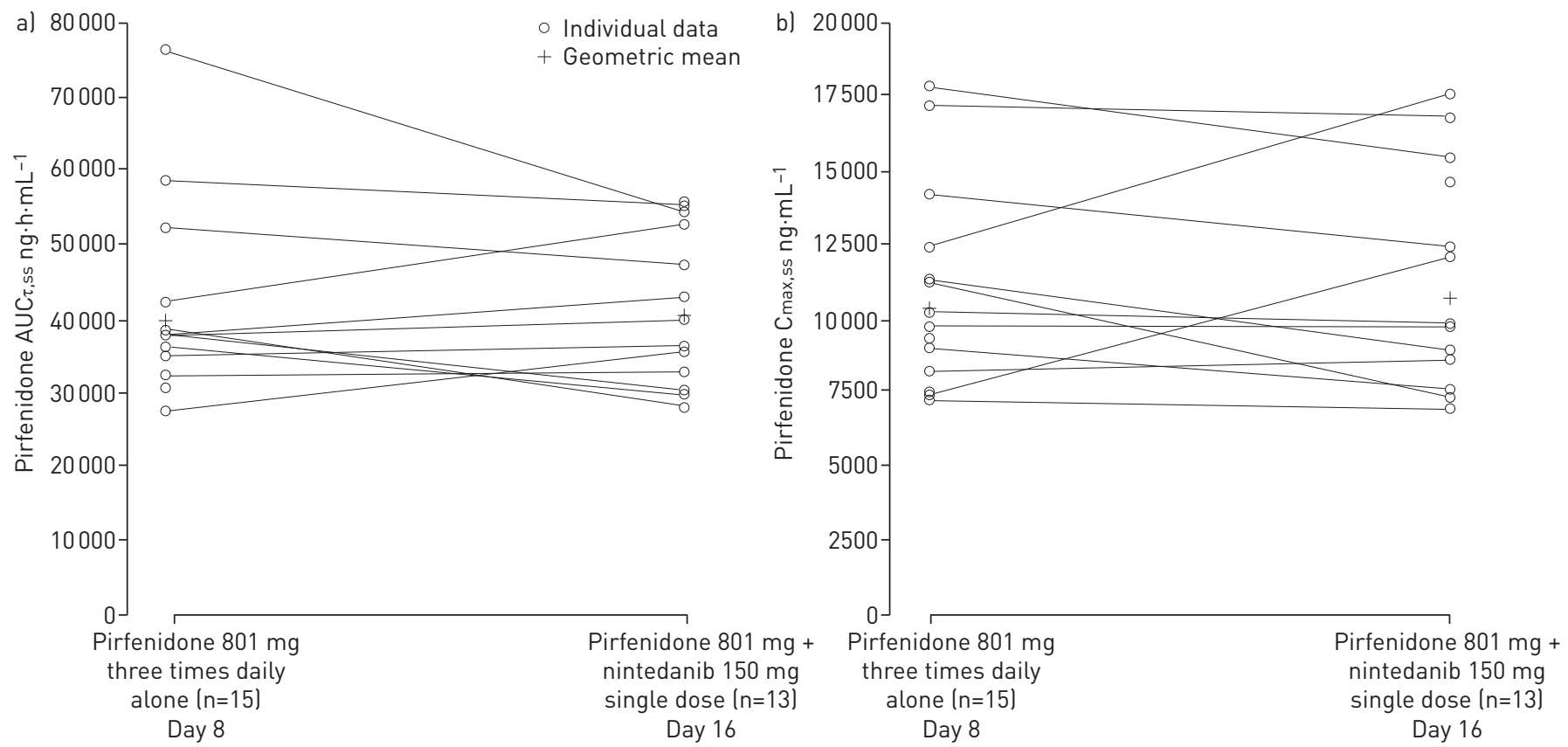

FIGURE 4 Comparison of individual and geometric mean (gMean) pirfenidone a) area under the concentration-time curve in plasma at steady-state over a uniform dosing interval $\tau\left(A \cup C_{\tau, s s}\right)$ and b) maximum concentration in plasma at steady-state ( $C_{\text {max,ss }}$ ) after oral administration of pirfenidone $801 \mathrm{mg}$ thrice daily alone, and together with nintedanib $150 \mathrm{mg}$ twice daily. 


\begin{tabular}{|c|c|c|}
\hline \multicolumn{3}{|l|}{ TABLE 4 Adverse events } \\
\hline & Group 1 & Group 2 \\
\hline Subjects & 20 & 17 \\
\hline Any adverse event(s) & $14(70.0)$ & $15(88.2)$ \\
\hline Severe adverse events & $2(10.0)$ & 0 \\
\hline Investigator-defined drug-related adverse events & $12(60.0)$ & $7(41.2)$ \\
\hline Adverse events leading to permanent dose reduction of pirfenidone & $1(5.0)$ & 0 \\
\hline Adverse events leading to discontinuation of nintedanib & 0 & 0 \\
\hline Adverse events leading to discontinuation of pirfenidone & $3(15.0)$ & 0 \\
\hline Other significant adverse events & $3(15.0)$ & 0 \\
\hline Serious adverse events ${ }^{\pi}$ & 0 & 0 \\
\hline \multicolumn{3}{|l|}{ Adverse events of particular interest } \\
\hline Diarrhoea $^{+}$ & $6(30.0)$ & 4 (23.5) \\
\hline $\mathrm{Nausea}^{+}$ & $5(25.0)$ & $3(17.6)$ \\
\hline Vomiting $^{+}$ & $3(15.0)$ & 2 (11.8) \\
\hline Bleeding & 0 & 0 \\
\hline
\end{tabular}

Data are presented as $\mathrm{n}$ or $\mathrm{n}(\%) .{ }^{*}$ : in treated population. ": adverse events reported for the on-treatment period. There was one fatal serious adverse event in the post-study period which was not considered to be drug-related. ${ }^{+}$: the Common Terminology Criteria for Adverse Events grading was assessed for each event.

Institute (NCI) Common Terminology Criteria for Adverse Events (CTCAE) grade 1. A total of 19 (51.4\%) subjects had possible drug-related adverse events. Eight of these individuals experienced diarrhoea and seven subjects had nausea. Dyspepsia, headache and fatigue were experienced by a further four subjects each, and three individuals reported vomiting. Three subjects permanently discontinued pirfenidone treatment due to adverse events (diarrhoea $n=2$; vomiting $n=1$; asthenia $n=1$ subject). No serious adverse events were reported.

\section{Discussion}

The approval of two novel antifibrotic drugs, pirfenidone and nintedanib, has transformed the management of IPF in recent years. As we search for further improvements in clinical outcomes in this patient population, nintedanib and pirfenidone combination therapy is an attractive therapeutic option to consider in view of their availability and differing mechanisms of action [7], but to date little is known about the potential for unanticipated drug-drug interactions or additive/synergistic efficacy or adverse events.

The results of the present study suggest that there is no relevant pharmacokinetic drug-drug interaction between nintedanib and pirfenidone in patients with IPF. In the primary analysis of group 1, gMean values of nintedanib exposure (based on AUC0-tz and Cmax) decreased by $12-19 \%$ when nintedanib was co-administered with pirfenidone. However, the primary analysis used data from all 17 subjects with evaluable pharmacokinetic observations to estimate the gMean ratios, and included five individuals who had high exposure values in the reference treatment period (with no noteworthy demographic characteristics), but no value for the test treatment. The sensitivity analysis that only included data from the 12 subjects with evaluable pharmacokinetic observations in both treatment periods resulted in gMean ratios of total and peak exposure that were close to $100 \%$. This suggests that the slight decrease in exposure of nintedanib observed in the primary analysis was primarily due to the unbalanced pharmacokinetic comparison combined with its known high inter-patient pharmacokinetic variability [24, 25].

Visual inspection of the individual nintedanib exposure values (AUC0-tz and $\mathrm{Cmax}$ ) provides further support for a lack of a pharmacokinetic drug-drug interaction. There was no obvious pattern for change in exposure between the test and reference treatments, and variability (both intra- and especially inter-individual) was high in the both groups. The pronounced inter-individual variability was also observed in other nintedanib studies [24, 25], including a prior trial of Japanese patients with IPF [18]. In the study enrolling Japanese patients, the pharmacokinetic assessment, due to the parallel-group design, was hampered by the inter-patient variability and, thus, did not provide a definitive conclusion about the effect of pirfenidone co-treatment on nintedanib exposure.

In group 2, pirfenidone exposure (based on $\mathrm{AUC}_{\tau, \mathrm{ss}}$ and $\mathrm{Cmax,ss}$ ) was similar whether pirfenidone was administered with or without steady-state nintedanib. The $90 \%$ confidence intervals were within the standard $80-125 \%$ bioequivalence acceptance range and the sensitivity analysis supported the primary 
analysis findings. The results for pirfenidone were consistent with the results from the previously described trial in Japanese IPF patients [18].

Supportive information about the drug-drug interaction of nintedanib and pirfenidone in IPF patients has recently been generated in the 12-week INJOURNEY trial. This trial focused on safety and tolerability, and only included sparse pharmacokinetic sampling [9]. Similar nintedanib trough plasma concentrations were observed when it was administered alone or with add-on pirfenidone.

Because the adverse reaction profiles of nintedanib and pirfenidone partially overlap, especially in terms of gastrointestinal events and liver enzyme elevations [12,15], there is a potential for additive adverse effects when combining both treatments. Nintedanib and pirfenidone were well tolerated in this study when administered alone and when co-administered. Most gastrointestinal events were of mild to moderate intensity and similarly distributed between the two treatment groups. Overall, $23(62.2 \%)$ out of 37 subjects across the two groups reported diarrhoea, nausea or vomiting events (all of which were classified as NCI-CTCAE grade 1), which was similar to that reported in previous studies $[9,18]$.

While the present data and previous studies $[9,18]$ suggest that there is no relevant pharmacokinetic drug-drug interaction between nintedanib and pirfenidone when co-administered in patients with IPF, further research is recommended to address some of limitations of the existing studies. Firstly, it may be informative to examine the activity of pirfenidone's major metabolite 5-carboxy-pirfenidone, as available in vitro data suggest some pharmacologically relevant activity of this compound at concentrations in excess of peak plasma concentrations in IPF patients [12]. Secondly, this study investigated in group 1 the effect of pirfenidone as the perpetrator drug at steady-state on the pharmacokinetics of nintedanib, the victim drug, after a single dose rather than at steady-state. However, as there was no clinically relevant drug-drug interaction in this "worst case scenario", it is strongly assumed that this finding similarly applies to steady-state dosing of nintedanib (the victim drug), as supported by the pharmacokinetic findings from the INJOURNEY study [9]. Thirdly, although data on the long-term use of nintedanib and pirfenidone monotherapy in patients with IPF are available $[26,27]$, there are currently only very limited data on the chronic use of combination therapy. A small phase II study in 20 Japanese patients with IPF has reported successful long-term treatment with nintedanib and pirfenidone together; the mean $\pm \mathrm{SD}$ duration of exposure in the extension was $27.0 \pm 15.8$ months and the safety and tolerability profile remained in line with the adverse event profiles for each drug, with no new safety signals identified [28]. In addition, concomitant treatment with nintedanib and pirfenidone for 12 weeks in the INJOURNEY trial was feasible with no new safety signals emerging [9]. However, no definite conclusions on the safety and tolerability of combination treatment can be drawn based on the small number of patients in these studies, and larger and longer trials investigating the long-term safety and tolerability of nintedanib given in addition to pirfenidone are required.

In conclusion, these data indicate that there is no relevant pharmacokinetic drug-drug interaction between nintedanib and pirfenidone when administered as combination therapy in patients with IPF. The plasma concentrations of the two drugs were similar when administered alone or in combination. The safety results of this trial were also consistent with the known adverse event profiles of the individual drugs.

Acknowledgements: This study was sponsored by Boehringer Ingelheim (Bracknell, UK) and was conducted at nine centres in the UK. The coordinating investigator was L. Richeldi, University of Southampton, Southampton, UK (since March 2017, Università Cattolica del Sacro Cuore, Rome, Italy). S. Fletcher, N. Chaudhuri and T.M. Maher acknowledge the support of the United Kingdom National Institute for Health Research (NIHR) Translational Research Collaboration for Inflammatory Respiratory Disease. Boehringer Ingelheim was responsible for the design and conduct of the study, and the collection and management of the data. Medical writing assistance, in the form of the preparation and revision of the manuscript, was supported financially by Boehringer Ingelheim and provided by Jonathan Brennan (Meditech Media, Manchester, UK) under the authors' conceptual direction and based on feedback from the authors. The authors were responsible for the study design, analysis and interpretation of the data and preparation of the manuscript. T.M. Maher is supported by an NIHR Clinician Scientist Fellowship (NIHR Ref: CS-2013-13-017) and a British Lung Foundation Chair in Respiratory Research (C17-3).

Conflict of interest: L. Richeldi reports grants and personal fees (member of an advisory board) from InterMune, personal fees for consultancy from Sanofi-Aventis, ImmuneWorks, Celgene, Nitto and Bristol Myers Squibb, personal fees as member of an advisory board from Roche, Fibrogen and Promedior, personal fees for speaking from Shionogi, personal fees as a member of a steering committee from Boehringer Ingelheim, personal fees for editorial activity from DynaMed, outside the submitted work. S. Fletcher has nothing to disclose. H. Adamali has nothing to disclose. N. Chaudhuri reports grants (for a project) from Boehringer Ingelheim, personal fees (for speakers fees) from Boehringer Ingelheim, other funding for educational sponsorship from Roche and Boehringer Ingelheim, and personal fees (for advisory boards) from Roche, outside the submitted work. S. Wiebe is an employee of Boehringer Ingelheim Pharma GmbH \& Co. KG. S. Wind is an employee of Boehringer Ingelheim Pharma GmbH \& Co KG. K. Hohl has nothing to disclose. A. Baker is an employee of Boehringer Ingelheim. R. Schlenker-Herceg has nothing to disclose. S. Stowasser is an employee of Boehringer Ingelheim International GmbH. T.M. Maher has, via his institution, received industry-academic funding from GlaxoSmithKline R\&D and UCB and has received consultancy or speakers fees 
from Apellis, AstraZeneca, Bayer, Biogen Idec, Boehringer Ingelheim, Cipla, GlaxoSmithKline R\&D, ProMetic, Roche, Sanumed and UCB.

Support statement: This study was supported by funding from Boehringer Ingelheim. Funding information for this article has been deposited with the Crossref Funder Registry.

\section{References}

1 Raghu G, Remy-Jardin M, Myers JL, et al. Diagnosis of idiopathic pulmonary fibrosis. An official ATS/ERS/JRS/ ALAT clinical practice guideline. Am J Respir Crit Care Med 2018; 198: e44-e68.

2 Martinez FJ, Collard HR, Pardo A, et al. Idiopathic pulmonary fibrosis. Nat Rev Dis Primers 2017; 3: 17074.

3 Raghu G. Idiopathic pulmonary fibrosis: lessons from clinical trials over the past 25 years. Eur Respir J 2017; 50: 1701209.

4 Noble PW, Albera C, Bradford WZ, et al. Pirfenidone in patients with idiopathic pulmonary fibrosis (CAPACITY): two randomised trials. Lancet 2011; 377: 1760-1769.

5 Richeldi L, du Bois RM, Raghu G, et al. Efficacy and safety of nintedanib in idiopathic pulmonary fibrosis. $N$ Engl J Med 2014; 370: 2071-2082.

6 Raghu G, Rochwerg B, Zhang Y, et al. An official ATS/ERS/JRS/ALAT clinical practice guideline: treatment of idiopathic pulmonary fibrosis. an update of the 2011 clinical practice guideline. Am J Respir Crit Care Med 2015; 192: e3-19.

7 Maher TM. Combination therapy and the start of a new epoch for idiopathic pulmonary fibrosis? Am J Respir Crit Care Med 2018; 197: 283-284

8 Wuyts WA, Antoniou KM, Borensztajn $\mathrm{K}$, et al. Combination therapy: the future of management for idiopathic pulmonary fibrosis? Lancet Respir Med 2014; 2: 933-942.

9 Vancheri C, Kreuter M, Richeldi L, et al. Nintedanib with add-on pirfenidone in idiopathic pulmonary fibrosis Results of the INJOURNEY trial. Am I Respir Crit Care Med 2018; 197: 356-363.

10 Richeldi L, Collard HR, Jones MG. Idiopathic pulmonary fibrosis. Lancet 2017; 389: 1941-1952.

11 Varone F, Montemurro G, Macagno F, et al. Investigational drugs for idiopathic pulmonary fibrosis. Expert Opin Investig Drugs 2017; 26: 1019-1031.

12 European Medicines Agency. Esbriet (Pirfenidone): Summary of Product Characteristics. www.ema.europa.eu/ documents/product-information/esbriet-epar-product-information_en.pdf Date last accessed: November 302017. Date last updated: October 312017.

13 Didiasova M, Singh R, Wilhelm J, et al. Pirfenidone exerts antifibrotic effects through inhibition of GLI transcription factors. FASEB J 2017; 31: 1916-1928

14 Conte E, Gili E, Fagone E, et al. Effect of pirfenidone on proliferation, TGF- $\beta$-induced myofibroblast differentiation and fibrogenic activity of primary human lung fibroblasts. Eur J Pharm Sci 2014; 58: 13-19.

15 European Medicines Agency. Ofev (Nintedanib): Summary of Product Characteristics. https://www.ema.europa.eu/ documents/product-information/ofev-epar-product-information_en.pdf Date last accessed: November 302017. Date last updated: September 192017.

16 Wollin L, Wex E, Pautsch A, et al. Mode of action of nintedanib in the treatment of idiopathic pulmonary fibrosis. Eur Respir J 2015; 45: 1434-1445.

17 King TE Jr, Bradford WZ, Castro-Bernardini S, et al. A phase 3 trial of pirfenidone in patients with idiopathic pulmonary fibrosis. N Engl J Med 2014; 370: 2083-2092.

18 Ogura T, Taniguchi H, Azuma A, et al. Safety and pharmacokinetics of nintedanib and pirfenidone in idiopathic pulmonary fibrosis. Eur Respir J 2015; 45: 1382-1392.

19 European Medicines Agency. Committee for Medicinal Products for Human Use (CHMP) Assessment Report for Esbriet (Pirfenidone). www.ema.europa.eu/docs/en_GB/document_library/EPAR_-_Public_assessment_report/ human/002154/WC500103073.pdf Date last accessed: November 30 2017. Date last updated: December 162010.

20 Stopfer P, Rathgen K, Bischoff D, et al. Pharmacokinetics and metabolism of BIBF 1120 after oral dosing to healthy male volunteers. Xenobiotica 2011; 41: 297-311.

21 European Medicines Agency. Committee for Medicinal Products for Human Use (CHMP) Assessment Report for Ofev (nintedanib). www.ema.europa.eu/docs/en_GB/document_library/EPAR_-_Public_assessment_report/human/ 003821/WC500182476.pdf Date last accessed: November 30 2017. Date last updated: November 202014.

22 European Medicines Agency. Committee for Human Medicinal Products (CHMP). Guideline on the Investigation of Bioequivalence. CPMP/EWP/QWP/1401/98 Rev. 1. www.ema.europa.eu/docs/en_GB/document_library/ Scientific_guideline/2010/01/WC500070039.pdf Date last accessed: November 30 2017. Date last updated: January 20,2010

23 Chow S, Liu J. Design and Analysis of Bioavailability and Bioequivalence Studies. New York, Marcel Dekker, 1992.

24 Eisen T, Shparyk Y, Macleod N, et al. Effect of small angiokinase inhibitor nintedanib (BIBF 1120) on QT interval in patients with previously untreated, advanced renal cell cancer in an open-label, phase II study. Invest New Drugs 2013; 31: 1283-1293.

25 Dallinger C, Trommeshauser D, Marzin K, et al. Pharmacokinetic properties of nintedanib in healthy volunteers and patients with advanced cancer. J Clin Pharmacol 2016; 56: 1387-1394.

26 Cottin V, Maher T. Long-term clinical and real-world experience with pirfenidone in the treatment of idiopathic pulmonary fibrosis. Eur Respir Rev 2015; 24: 58-64

27 Brunnemer E, Wälscher J, Tenenbaum S, et al. Real-world experience with nintedanib in patients with idiopathic pulmonary fibrosis. Respiration 2018; 95: 301-309.

28 Taniguchi $\mathrm{H}$, Ogura $\mathrm{T}$, Inoue $\mathrm{Y}$, et al. Long-term safety of combination therapy with nintedanib and pirfenidone in Japanese patients with IPF. Eur Respir J 2016; 48: Suppl. 60, PA2089. 